\title{
'Nobody ever chooses this ...': a qualitative study of service providers working in the sexual violence sector - key issues and challenges
}

\author{
Caroline Kelleher BA and Sinéad McGilloway BSSc, PhD, CPsychol, CSci, AFBPS
}

Department of Psychology, National University of Ireland Maynooth, Ireland

\author{
Correspondence \\ Caroline Kelleher \\ Department of Psychology \\ NUI Maynooth \\ Maynooth \\ Co. Kildare, Ireland \\ E-mail: caroline.kelleher@nuim.ie
}

\begin{abstract}
This paper describes the findings from the first stage of a study designed to assess the impact of sexual violence (SV) on the lives of Irish women. Stakeholder interviews $(n=18)$ were undertaken to explore experiences of delivering services to people who have experienced SV, in rural and urban settings in Ireland. A range of themes and sub-themes were identified through a thematic analysis, and interrelationships between two of the main themes (barriers to services and current gaps in service provision) are discussed. Sub-themes include: survivor guilt and shame, difficulties in naming the incident as SV, problems with the Criminal Justice System and the availability of Sexual Assault Treatment Units. Participants identified an urgent need for a co-ordinated national strategy in the form of a public awareness and education campaign in order to undermine commonly held rape myths and stereotypes. The findings will be of interest to all health and social care professionals who are in contact with women who have experienced SV. They will also help to raise an awareness of the 'hidden' costs of SV, and inform appropriate policies and community-based responses to this important social problem.
\end{abstract}

Keywords: rape, service providers, service provision, sexual violence

Accepted for publication 14 October 2008

\section{Introduction}

Research suggests that one in every three women has been assaulted, coerced or been a victim of some form of sexual abuse during their lifetime (Heise et al. 1999, Krug et al. 2003, Tavara 2006, p. 397). However, it is widely acknowledged that rape is one of the most under-reported of all crimes (Crowell \& Burgess 1996, Tjaden \& Thoennes 2006). The impact of sexual violence (SV) on the lives of young women in Ireland is unknown, despite the substantial increase in reported sexual offences in Ireland since 1950 (Young et al. 2001). Results from the Sexual Abuse and Violence in Ireland (SAVI) report - the first prevalence study on SV conducted in Ireland - found that $42 \%$ of women have experienced sexual abuse or assault in their lifetime (McGee et al. 2002 , p. 120). Furthermore, $47 \%$ had never told anyone prior to participating in the study (McGee et al. 2002, p. 121). While these figures are broadly consistent with those from elsewhere, Ireland has the lowest conviction rate (1-2\% during 1993-2000) for rape and sexual assault in Europe (Regan \& Kelly 2003, p. 10).

Victims, or survivors, of SV (both terms are used interchangeably here) experience numerous negative effects on their lives including: resource disruption (e.g. unemployment, divorce) (Monnier et al. 2002); deterioration in interpersonal functioning (Schumm et al. 2004); elevated risk of suicide (Ullman 2004); and an increased utilization of medical services (Stein et al. 2004). These findings have important implications for health and social services, and for informing the development of effective treatments and interventions. In Ireland, the Rape Crisis Network of Ireland (RCNI) is the national umbrella organization for the 16 community-based Rape Crisis Centres (RCCs) that respond to the needs of those who have experienced SV (RCNI 2005). Each centre operates independently and offers a range of services including: one-to-one crisis counselling, long-term counselling, accompaniment to Sexual Assault Treatment Units (SATUs) and court appearances and advocacy 
and information. Several centres also provide community outreach services, education and training and community awareness-raising initiatives. All RCCs rely heavily on fundraising in order to sustain and develop their services. They vary with regard to number of staff, opening hours and phone line operating hours (where applicable). The staff that work in RCCs typically include a director of the service, counselling therapists and various administration staff. In smaller centres, one person, often the director, frequently fills multiple roles. With regard to the SATUs, four currently operate in Ireland - in Dublin (east), Cork (south), Waterford (south-east) and Letterkenny (north-west). They offer forensic and/or medical services within local or national hospitals, but vary widely with respect to funding, management, staffing and scope of service provision (O'Shea 2006). The Cork-based SATU is the only one currently in receipt of statutory funding.

The Dublin Rape Crisis Centre (DRCC) (the largest in Ireland) operates the only available 24-hour help line in the country; almost 16000 calls were received in 2006, approximately three-quarters of which were genuine counselling calls (DRCC 2007, p. 11). Most callers were female ( $84 \%)$ and over half (55\%) involved adult rape and sexual assault, representing an increase of $3.5 \%$ since $2005(2007$, pp. 6,14$)$. In $60 \%$ of cases the perpetrator was known to the victim. During 2007, DRCC volunteers accompanied 315 victims to the Dublinbased SATU (an increase of $20 \%$ since 2005) (p. 6). Additionally, DRCC therapists delivered 3585 individual client sessions, $35 \%$ of which involved crisis appointments for men and women who had experienced rape and sexual assault during the previous 12 months (DRCC 2007, p. 14).

The present study forms part of a larger, multistage, mixed method investigation designed to describe and explore the impact of SV on the lives of Irish women. As far as we are aware, no peer-reviewed qualitative studies of service providers in this area have been conducted within Europe, while few have been conducted farther afield. The qualitative methodology used here is well suited to the topic under investigation in view of its sensitive and relatively novel nature (Patton 2002). The key objectives of this study were to: (1) identify key service providers from national representative organizations; (2) obtain detailed information on the extent and nature of current service provision; and (3) ascertain the key issues and challenges in this area from the perspective of service providers.

\section{Subjects and methods}

An initial scoping survey was conducted to identify key stakeholders throughout Ireland who were working in the SV sector. Individual staff from 24 organizations were contacted, 18 of whom agreed to participate in one-to-one interviews. All of the interviews were conducted on a face-to-face basis by the first author (C.K.). Participants had variable levels of experience and worked in a range of urban and rural services including RCCs, or other SV services $(n=14)$, SATUs $(n=3)$ and domestic violence agencies $(n=1)$.

A semistructured interview schedule was devised following an extensive review of the literature. The items, some of which were organization specific, inquired about services provided, staffing arrangements, routinely available data, perceived gaps in current service provision and the requirements for high-quality services. The interview schedule was piloted with the first few participants, with only minimal modifications. Prior to the interview, participants received an information sheet and, upon request, a sample of the interview topics. They also completed a brief background questionnaire. Interviews lasted from 45 minutes to 2 hours, and all were audio-recorded with consent (excluding four who declined) and transcribed (by C.K.) in preparation for a thematic analysis (Hayes 2000). The transcripts were judged to accurately reflect the content of the interviews. Each interview was read and re-read several times, after which all items of interest were noted and colour coded by hand, and organised into proto-themes which were carefully examined and defined. The interview schedule was used as an initial 'theme guide' to organizing this material. A secondary examination of the transcripts was then conducted, and material relevant to each theme was identified, colour coded and organised into a systematic coding scheme. It was then possible to construct each theme in its final form which consisted of a name, definition and supporting data. The four interviews that were not recorded were examined only after the 14 other transcriptions had been analysed; any new material was included in the final analysis in the same iterative fashion. A random sample of the transcripts was checked by an independent researcher for purposes of validating the key themes and sub-themes.

\section{Results}

\section{Participant profile}

All participants $(n=18)$ were women ranging in age from 30 to 59 years, and had worked in the SV sector for an average of 8.5 years. They included counselling therapists $(n=10)$, nurses $(n=2)$, a doctor/consultant $(n=1)$ and managers/administrators $(n=5)$. Half of the sample rated current service supports for women who have experienced SV as only 'fair' to 'poor', while $90 \%$ reported the amount of stigma associated with this 
Table 1 List of themes and sub-themes including descriptions

\begin{tabular}{|c|c|}
\hline Theme name & Description of sub-theme \\
\hline \multicolumn{2}{|l|}{ Theme 1: Barriers to Services } \\
\hline 1.1 Survivors' shame and guilt & Feelings of guilt and shame post-assault act as a barrier to survivors in accessing services \\
\hline $\begin{array}{l}\text { 1.2 Naming/Acknowledgement of } \\
\text { the incident }\end{array}$ & $\begin{array}{l}\text { Victim reluctance to name the incident as sexual violence (SV) means they fail to } \\
\text { identify themselves as eligible for services }\end{array}$ \\
\hline 1.3 Societal myths around rape & Persistent societal myths around rape facilitate further self-blame in victims \\
\hline \multicolumn{2}{|l|}{ Theme 2: Current Gaps in Service Provision } \\
\hline 2.1 Education and awareness & Lack of awareness around services, and the prevalence and effects of SV \\
\hline 2.2 The Criminal Justice System (CJS) & Gaps exist in the CJS which can affect the likelihood of a woman proceeding with a case \\
\hline 2.3 Availability of Sexual Assault & Issues around the geographic spread of SATU services and the operation \\
\hline Treatment Units (SATUs) & of a 'true' 24-hour service \\
\hline
\end{tabular}

crime to be considerable. Two key themes were identified and/or emerged from the analysis, within which a further six sub-themes were identified. These are described and summarised in Table 1.

\section{Barriers to service provision}

Survivors' shame and guilt. A powerful and recurring theme - and one mentioned by all participants - related to the shame and guilt felt by many women after being raped or sexually assaulted; this appears to pose one of the most significant barriers to accessing appropriate support and reporting to the police. For example, one participant commented:

... I think the main difficulty ... is the whole stigma and shame ... It's a combination of stigma and shame, and a lack of awareness among women of what sexual assault actually is ... (RCC service provider, 4 years of experience)

Participants also alluded to the negative experiences of disclosure among some women and how this may compound existing levels of guilt. If women feel a sense of responsibility for the incident, the effects of disclosing to someone who reinforces these beliefs, may prevent them from disclosing in the future, or from identifying themselves as in need of support services. Two participants commented:

... Quite a lot of clients will self-blame ... the clients that come in here still feel that they are to blame for what has happened even though they know ... part of their brain is telling them ... 'No, he was wrong'... (SATU service provider, 2 years of experience)

... They definitely have a huge guilt thing that you know ... 'God, was it something I did?' ... (SATU service provider, 5 years of experience)

Feelings of shame, guilt and self-blame are common following an experience of SV and frequently give victims some sense of control over their world (Petrak
\& Hedge 2002). Issues perceived by respondents to present important obstacles to women when accessing formal or informal support included: the shame related to their perceived responsibility for the attack; how much they resisted it; and concerns about the attitudes of friends and family. Unless these kinds of issues can be addressed at an individual and societal level, essential support services will remain underutilised and underfunded into the future.

Naming/Acknowledgement of the incident. In order for women to see themselves in need of, or suitable for, the services provided by RCCs or SATUs, they must first recognise that what they have experienced is SV and a crime. This naming or acknowledgement of the incident was another recurring sub-theme; for example, one participant reported:

... for a lot of women who have experienced unwanted sexual contact or, coercion into having sex ... they may not name that as rape ... (RCC service provider, 12 years of experience)

This reluctance to name what has happened to them often leads to a sustained silence during which women struggle with many negative emotions. This may have serious implications for service providers who are trying to reach out to vulnerable women. Thus, service providers need to balance their desire to inform women of their services (through the use of appropriate language, images and media) with their need to ensure that they do not alienate an already marginalised and vulnerable population.

Societal myths around rape. Several participants alluded to the numerous rape myths that still persist in today's society and, in particular, the prevailing stereotypes about who is a 'victim' and who is a 'perpetrator'. The stereotypical 'rape victim' is illustrated in the following: 
... an awful lot of people won't ... don't identify with that word [rape] because they see it as... Well, rape means someone dragging you down an alleyway, sticking a knife to your throat and beating the crap outta ya!... (Domestic violence service provider, 12 years of experience)

The consequences of experiencing SV outside of these parameters often lead to a high degree of self-blame among women; as a result, they tend to feel that they are not entitled to support services, or even justice. The following highlights some of the typical misconceptions that women must overcome in order to access services:

... We have a notion in this society that rape is a stranger who jumps out of a bush when a woman is walking home. Certainly, she doesn't know him, she doesn't go on a date with him, she never kissed him and she's NOT married to him ... so, that makes it much more difficult for women who are raped in those circumstances ... (RCC service provider, 13 years of experience)

The potential likelihood that a woman will not be believed by those around her is often a significant barrier to disclosure and subsequent service utilization, but this is perhaps no more salient than when the rape or sexual assault occurs within an intimate partnership or marriage:

... Well, you're in the relationship with him so you must have said something then to provoke this! ... It's really difficult for women to feel that they're going to be taken seriously and that they're going to be believed ... (RCC service provider, 2 years of experience)

This finding is consistent with quantitative research that suggests that marital rape is experienced by about $10-14 \%$ of all married women, and by about $40-50 \%$ of battered women (Martin et al. 2006, p. 337). These kinds of misinformed attitudes may extend beyond a woman's immediate family circle and close friendships, and may have consequences in many areas including the treatment of women within the Criminal Justice System (CJS). The potential difficulties with the CJS and its treatment of victims of SV are explored in more detail below.

\section{Current gaps in service provision}

Education and awareness. Participants repeatedly stressed the need for, and the importance of, education and awareness raising. Some commented that several women they had encountered either directly through their service, or external to it, had been unaware of their local services. One participant noted:

... I mean people say to me when I say to them I work in the Rape Crisis Centre ... they say I didn't know it even existed ... there has to be an awareness around counselling ... that it works, that people need counselling ... (RCC service provider, 5 years of experience)
Several participants mentioned the tensions inherent in promoting their service while ensuring a safe, confidential and therapeutic 'space' for women - in other words, providing a place of sanctuary where women felt sufficiently secure to confide in someone. For example, one RCC worker felt that, if more women were accessing the service, some might feel more easily identifiable when entering and exiting the RCC premises, thereby compromising their confidentiality. Nonetheless, a therapeutic 'space' that is safe physically, emotionally and psychologically was considered by most RCC workers to be essential to their clients' recovery. However, all were also aware of the need for public education initiatives:

... One of the biggest things is education and information ... I think that teaching young people about consent is absolutely essential ... What does consent mean? ... How do you know if someone is consenting? ... Do they have to say 'yes' or 'no'? ... (RCC service provider, 6 years of experience)

Participants were clear about the need to increase awareness of the seriousness and impact of SV, and an understanding of the difficulties faced by women when trying to access support. Common responses to victims of SV were seen by one participant as being particularly unhelpful:

... What was she wearing? ... Why was she there? ... Why did she go with him? ... that's a natural thing [to ask those questions] but in some ways, it's a societal thing... it has to be seen that ... nobody ever chooses this [rape] ... (RCC service provider, 12 years of experience)

Worryingly, wider society was considered by some participants to be characterised by a 'rape-supportive' culture. It was suggested that without effective national education and awareness raising programmes - similar to the 'Don't Drink and Drive' campaigns run by the Irish Road Safety Authority and the Department of Transport - women who experience SV will have to continue to decipher mixed messages and information before accessing services. One participant commented:

... there's a huge gap in services ... how is it that people know first of all that sexual violence is wrong because we [society] don't hand out that message ... (RCC service provider, 13 years of experience)

The CJS. Several interviewees expressed disappointment and frustration with the CJS and particularly the manner in which women are treated if they proceed with a case. Our findings suggest that the experience for women who intend to prosecute the alleged perpetrator is at best stressful, and at worst, re-victimizing. Participants expressed particular frustration with the length of waiting time for a case to come to court, and with the treatment of the victim as a 'state witness'. This can often mean that the victim is not always kept informed of developments in the case. As one participant reported: 
... the biggest stumbling block in rape cases is the justice side of it from what I can gather ... like they're [rape victims] only witnesses ... (SATU service provider, 5 years of experience) (Note: The CJS is not legally obliged to keep a witness informed of progress in the case, although in many cases they try to.)

Another issue with the Irish CJS is the current Director of Public Prosecution's (DPP) policy, whereby there is no obligation to provide a reason for a decision to abandon a case. The decision by the victim to proceed with a case is, at best, a difficult one, but to have a case dropped for no reason must be even more frustrating. Remarkably, one participant whose job involved collecting the forensic evidence used in these cases, commented:

... You do hear things from people [about the CJS] ... that's where I'd see the biggest gap in the service ... not so much at this level [SATU], but I think from here on in, it's horrendous... maybe the fortunate ones are the ones that don't go to court ... (SATU service provider, 2 years of experience)

According to some participants, even when a case reaches court, the attitudes of CJS personnel may sometimes be far from understanding and supportive. Furthermore, the myths and stereotypes surrounding rape are not peculiar to the general public as illustrated by the following:

... the fourth one [court accompaniment by RCC volunteer] was just a 'not guilty' plea ... and just how that girl was treated by the judge, by the Guards [the Gardai - the Irish police force] ... you're kind of going 'Is this still the Dark Ages?'... (RCC service provider, 8 years of experience)

Availability of SATUs. An urgent need for an increase in the number of appropriate and accessible SATUs was highlighted throughout the interviews, as was the need to offer a 24-hour service aimed at meeting women's medical and forensic needs:

... the biggest [gap in services] ... is obviously the fact that the [SATU] services aren't available 24 hours ... what I would like to see is that the SATUs were more available ... (RCC service provider, 18 years of experience)

Some SATUs only provide services to victims if they are willing to proceed with a case and have a forensic medical examination (FME). Funding issues and difficulties in securing and retaining qualified doctors to carry out the FMEs also affect service availability. Several service providers, particularly from rural areas, spoke about women having to remain in the same clothes they had been wearing at the time of the assault, for up to 2 and 3 days afterwards, while waiting for a suitably qualified FME. The likely occurrence of this appears to be determined largely by a woman's proximity to a SATU. As one participant commented:
... the SATU ... we're quite far away from Dublin and that fact that we don't have the personnel to offer a 24-hour service ... there are four social workers who are really keen for a SATU unit to be put in XX [name withheld] ... that's not going to happen for a very long time ... (RCC service provider, 6 years of experience)

Further comments were made about the uneven geographical spread of SATU services within Ireland. According to a national review, SATU services in Ireland 'have developed in a relatively fragmented way rather than as a co-ordinated strategy' (O'Shea 2006, p. 39). Consequently, a large portion of the population is under-served. One service provider remarked:

... I think if you look at the [SATU] services ... in general in the West of Ireland, in the Midlands and in the Northern part of the country, the border part of the country ... they are a scandal ... (SATU service provider, 16 years of experience)

Many of these issues require an urgent response, while others, such as public attitudes and behavioural change, will require a more carefully constructed and co-ordinated, inter-agency approach in the longer term. The findings are discussed in more detail as follows.

\section{Discussion}

A key strength of this study was its qualitative approach and its emphasis on service providers. Previous small-scale research undertaken in Ireland has identified some of the issues in the SV sector, but only within the context of victims' experiences of the judicial system and a national review of sexual assault services based largely on documentary data (Leane et al. 2001, O'Shea 2006). The use of qualitative methods in the current study provides useful insights into the key issues affecting victims and the experiences of service providers. The results suggest a distinct, yet subtle interplay between the themes and sub-themes. For example, is the shame and guilt that women feel after an experience of SV influenced by societal rape myths? Are the barriers identified here in some way related to the gaps in existing services? These and other questions require careful examination in the context of the present findings.

\section{The role of rape myths}

Rape myths have been defined as 'prejudicial, stereotyped or false beliefs about rape, rape victims and rapists' (Burt 1980, p. 1). Some examples of these-from both the current and other studies (Burt 1980) - include: 'women ask for it', 'all women secretly want to be raped' or 'women are only raped in dark alleys by a stranger'. A relatively recent UK telephone survey $(n=1095)$ (Amnesty International UK 2005) revealed some 
worrying trends in public views and understanding of rape/sexual assault. Almost one-third felt that, if a woman was intoxicated, she was in some way responsible for being raped (p. 7), while more than one in five felt that a woman would be 'partially' or 'totally' responsible if she was walking alone in a dangerous or deserted area (Amnesty International UK 2005, p. 6). A recent attitude poll conducted in Ireland $(n=1002)$ reported similar results (Irish Examiner/Red C 2008). These societal myths and attitudes contrast sharply with official statistics which indicate that only a minority of Irish women are assaulted in a public place or outdoors $(24 \%)$, and that the assault is more often carried out by someone they already know (79\%) (McGee et al. 2002, pp. 98, 101). The impact of these rape myths means that many women have difficulty in defining what has happened to them. Thus, Kahn et al. (2003, p. 233) reported that at least half of women who have been raped 'do not label themselves as rape victims'. Our findings suggest that this can pose a significant barrier to accessing services.

Rape myths have also been found to compound the feelings of self-blame and guilt experienced by women in the aftermath of SV. Results from several studies indicate that the responsibility for the rape (in a hypothetical scenario) is often attributed to the woman (e.g. Acock \& Ireland 1983, Renner et al. 1988). Furthermore, guilt and self-blame have been identified as strong reasons for non-reporting in female victims of acquaintance rape ( $n=236$ ) (Bachman 1998). Unsurprisingly, these feelings may affect a woman's decision to disclose, and it is interesting to note that $47 \%$ of the SAVI study sample had never told anyone about their experience of SV prior to taking part in the research (McGee et al. 2002, p. 121). Clearly, such internalised rape myths, in conjunction with a reluctance to name their experience as SV, may deter women from seeking help.

A small qualitative study $(n=8)$ by Ahrens (2002) found that some of the participants following negative interactions with service providers questioned whether they had actually experienced rape. Ahrens (2002) reported that these were rooted largely in rape myths and stereotypes. Participants who had initially disclosed their experience of SV to both formal (and informal) support providers proceeded to maintain their silence for some time after these interactions (Ahrens 2002). This delay in accessing support may, in turn, impact upon recovery from the effects of the assault and the knock-on consequences on their lives (Herman 2001). Interestingly, Sable et al. (2006) found that the concerns of women in the USA are still dominated by shame, guilt and embarrassment, despite 30 years of legal reforms and public education campaigns, and rape awareness activities on college campuses.

\section{Gaps in our services and gaps in our society}

Participants in this study referred to several important and persistent gaps, in the way society responds to victims of SV and in the inadequate provision of information/ education and rape-related services. Some of the legal issues highlighted here and relating to the treatment of rape victims within the Irish CJS have been documented in some small-scale research within Ireland (e.g. Bacik et al. 1998, Leane et al. 2001), although much less is known about the adequacy of available support services across sectors generally. The findings also highlight an important need to increase the availability of support services including, in particular, the SATUs.

Leane et al. (2001) identified several inadequacies within the Irish CJS including delays in rape cases reaching trial, low rates in convictions and a lack of understanding of the psychological impact of rape. Our findings suggest that there has been little progress in providing an effective and a compassionate legal response to victims of SV. Our participants were very explicit about some of these legal barriers and the often-negative, stereotypical attitudes of CJS personnel. The key legal actors with whom victims engage include the Garda (police), prosecution and defence lawyers and judges. Training has been recommended for all of these personnel in relation to rape myths, rape trauma syndrome and sensitivity to victims (Leane et al. 2001). Notably, the Cork Sexual Violence Centre has just launched a step-by-step guide to the legal system for both professionals and clients proceeding with a rape case (Crilly 2007). A statutory agency, entitled The National Office for the Prevention of Domestic, Sexual and Gender-based Violence (known as 'Cosc'), has also been set up recently to combat violence against women (Women's Health Council 2007). Cosc has already expressed a strong interest in the current study, and it is hoped that our findings will be used to inform the development of appropriate and responsive services within the Irish CJS and perhaps farther afield.

All four SATUs in Ireland face several challenges including: the recruitment and retention of trained FMEs, the sometimes conflicting roles of nursing staff to provide appropriate out-of-hours cover (e.g. one of the SATUs relies on nurses from the gynaecological ward of the adjacent hospital) and the limited scope of service provision (e.g. Waterford SATU services are only available to victims who proceed to court) (O'Shea 2006). Comparable challenges exist within the UK. For instance, Coy et al. (2007) reported that fewer than one in four local authorities have a specialised SV support service (p. 33). Furthermore, Northern Ireland does not have a Sexual Assault Referral Centre, while its only RCC lost its government funding in July 2006 (Coy et al. 2007). 
Thus, the geographical spread of services and unstable sources of funding, as in Ireland, mean that many regions are grossly under-served.

Since this study began, plans have been announced to establish two further SATUs in Ireland - one in the Midlands and a second in the west of the country (HSE 2007). The Irish government also announced funding of $€ 1.5$ million for SATUs for the remainder of 2007, and $€ 2.5$ million for every year thereafter (HSE 2007). These developments have come in timely response to a national review of sexual assault treatment services (O'Shea 2006). Nonetheless, our findings suggest that there is still considerable scope for improvement and for implementing some of the other key recommendations in the O'Shea report, such as standardizing existing services and commissioning needs-led research (O'Shea 2006). Unfortunately, the current downturn in the economy may pose an obstacle to implementing these recommendations and to ensuring that ring-fenced funding for the SV sector is provided into the future.

\section{'Prevention is better than cure': education and awareness raising}

Our findings are broadly consistent with the small pool of qualitative studies which have been conducted with counsellors/RCC staff in both the USA (O'Sullivan \& Carlton 2001, Logan et al. 2005) and Australia (e.g. Carmody 1997). They represent a timely contribution to tackling the important issue of SV and provide some important cues to action. There is a cautious, but growing awareness of this crime in Ireland and of its impact on women, their families, neighbourhoods and communities, as well as the demands on health and social services. However, as long as the blame continues to be placed largely at the feet of victims, the societal problem of SV will remain unacknowledged. Our findings suggest that a substantial shift is required in our collective thinking about rape (e.g. who is a perpetrator; who is a victim).

Recently, the World Health Organization (WHO) highlighted an urgent need for the primary prevention of SV, including violence that occurs within intimate relationships (Harvey et al. 2007). The concept of primary prevention, in the context of SV, means 'reducing the number of new instances of intimate-partner violence (IPV) or sexual violence by intervening before any violence occurs' (Harvey et al. 2007, p. 5). A growing body of literature purports that rape prevention education can elicit the cognitive, emotional and behavioural changes required to develop a 'rape consciousness' (Lonsway et al. 1998, Klaw et al. 2005). Participants in our study repeatedly emphasised a pressing need for education and awareness-raising initiatives to address negative stereotypes and rape myths; this is also underlined in six of the eight recommendations in the SAVI report (McGee et al. 2002). Recently, Fisher et al. (2008) examined the role of research in developing appropriate and effective rape prevention programmes and risk reduction interventions. They identified several key guiding concepts such as increasing awareness/knowledge about rape and reducing rape myths/rape supportive attitudes (Fisher et al. 2008). While some of the Irish RCCs run external education and training courses, these are, for the most part, not funded. Arguably, the need for more funding for extra staff should not be at the expense of explicit attempts at prevention, which should be funded solely by the Irish government (McGee et al. 2002).

Fortunately, this move towards primary prevention and awareness raising is already evident in certain service sectors both in Ireland and elsewhere. For example, the DRCC have just launched the first ever awarenessraising campaign (in conjunction with Cosc) and have commissioned a TV advertising and an outdoor poster campaign. Some of the issues in which Cosc has become involved include: ensuring the delivery of well-co-ordinated services for victims and raising awareness of the level and impact of these crimes and of local support services (http://www.cosc.i.e. 22/07/08). These developments suggest the beginnings of a stirring in our collective consciousness that will hopefully increase our knowledge of this crime and begin to eradicate some well-known (and ultimately harmful) myths and stereotypes.

Education initiatives targeted at multilevels of society are clearly needed to create a less hostile environment and culture, where victims of SV can safely seek help, pursue and secure justice and heal from the trauma of their experiences. Klaw et al. (2005, p. 61) assert that 'intensive, sustained rape education efforts play a vital role in dismantling rape supportive culture'. Notably, however, the WHO indicates that the complex behaviours associated with SV may not be irrevocably changed using public information campaigns in isolation (Harvey et al. 2007). Thus, a longer-term, multi-pronged approach may be required in order to properly tackle this problem.

\section{Conclusion}

SV against women needs to be strategically positioned on the national and international radar. An evidencebased, co-ordinated and inter-agency response is required to address the issues raised here and, in particular, to dismantle the persistent societal rape myths and improve service provision. This may be achieved through ongoing school-, community- and media-based education and 
awareness-raising initiatives. While progression in the area of rape prevention is clearly the next logical step, such endeavours must not be at the cost of providing adequate and well-resourced support services. In particular, there should be an increased focus on improving the experience of victims within the health and judicial systems. The process of addressing SV, while responding to the needs of victims, is a challenge that should perhaps begin with an acknowledgement of SV as a societal problem. Promoting ownership of the problem and implementing appropriate interventions and awarenessraising initiatives will help to foster a more supportive and understanding society in which victims of SV can feel more protected and secure.

Further research also ought to focus on the prevalence and impact of SV in men in Ireland, as well as SV within the context of marriage and / or intimate relationships. Importantly, the findings presented here have been used to inform the second and third stages of the study, which involve, respectively, a survey of women $(n \cong 80)$ who have experienced SV and a series of in-depth interviews with a smaller sub-sample. The collective findings should help to promote a better understanding of the impact of this trauma on the lives of a significant minority of women in Ireland and elsewhere.

\section{Acknowledgements}

We would like to thank all those who participated in this research. We also acknowledge the Irish Research Council for Humanities and Social Sciences who funded this study.

\section{References}

Acock A.C. \& Ireland N.K. (1983) Attributions of blame in rape cases: the impact of norm violation, gender and sex-role attitude. Sex Roles 9, 179-193.

Ahrens C.E. (2002) Silent and the silenced: the disclosure and non-disclosure of sexual assault. Dissertation Abstracts International: Section B: The Sciences and Engineering 63 (3-B), 1553-1561.

Amnesty International UK (2005) Sexual Assault Research Summary Report. ICM, London.

Bachman R. (1998) The factors related to rape reporting behaviour and arrest: new evidence from the National Crime Victimization Survey. Criminal Justice Behaviour 25, 829.

Bacik I., Maunsell C. \& Gogan S. (1998) The Legal Process and Victims of Rape: The Findings. Dublin Rape Crisis Centre, Dublin.

Burt M.R. (1980) Cultural myths and support for rape. Journal of Personality and Social Psychology 38 (2), 217-230.

Carmody M. (1997) Submerged voices: coordinators of sexual assault services speak of their experiences. Affilia 12 (4), 452-470.

Coy M., Kelly L. \& Foord J. (2007) Map of Gaps: The Postcode
Lottery of Violence Against Women Support Services. End Violence Against Women, London.

Crilly M. (2007) Sexual Violence in Ireland: The Criminal Justice System, a Guide for Victims. Cork Sexual Violence Centre, Cork.

Crowell N.A. \& Burgess A.W. (Eds) (1996) Understanding Violence Against Women. American Psychological Association, Washington, DC.

DRCC (2007) The Dublin Rape Crisis Centre Annual Report 2006. Dublin Rape Crisis Centre, Dublin.

Fisher B.S., Daigle L.E. \& Cullen F.T. (2008) Rape against women: what can research offer to guide the development of prevention programs and risk reduction interventions? Journal of Contemporary Criminal Justice 24, 163-177.

Harvey A., Garcia-Moreno C. \& Butchart A. (2007) Primary Prevention of Intimate-partner Violence and Sexual Violence: Background Paper for WHO Expert Meeting, May 2-3, 2007. World Health Organisation, Geneva.

Hayes N. (2000) Doing Psychological Research: Gathering and Analysing Data. Open University Press, Philadelphia, PA.

Health Service Executive (HSE) (2007) New resources for SATUs. Healthmatters 3 (3), 1-32.

Heise L., Ellsberg M. \& Gottmoeller M. (1999) Ending violence against women. Population Reports Series L, No. 11, 1-44.

Herman J.L. (2001) Trauma and Recovery. Pandora, London.

Irish Examiner/Red C (2008) Rape: Our Blame Culture. Published 26 March 2008.

Kahn A.S., Jackson J., Kully C., Badger K. \& Halvorsen J. (2003) Calling it rape: differences in experiences of women who do or do not label their sexual assault as rape. Psychology of Women Quarterly 27 (3), 233-242.

Klaw E.L., Lonsway K.A., Berg D.R., Waldo C.R., Kothari C., Mazurek C.J. \& Hegeman K.E. (2005) Challenging rape culture: awareness, emotion, and action through campus acquaintance rape education. Women $\mathcal{E}$ Therapy 28 (2), 47-63.

Krug E.G., Dahler L.L., Mercy J.A., Zwi A.B. \& Lozano R. (Eds) (2003) World Report on Violence and Health. World Health Organisation, Geneva.

Leane M., Ryan S., Fennell C. \& Egan E. (2001) Attrition in Sexual Assault Offence Cases in Ireland: A Qualitative Analysis. The Stationery Office, Government Publications, Dublin.

Logan T.K., Evans L., Stevenson E. \& Jordan C.E. (2005) Barriers to services for rural and urban survivors of rape. Journal of Interpersonal Violence 20 (5), 591-616.

Lonsway K.A., Klaw E.L. \& Berg D.R. (1998) Beyond 'no means no': outcomes of an intensive program to train peer facilitators for campus acquaintance rape education. Journal of Interpersonal Violence 13 (1), 73-92.

Martin E.K., Taft C.T. \& Resick P.A. (2006) A review of marital rape. Aggression and Violent Behaviour 12 (3), 329-347.

McGee H., Garavan R., de Barra M., Byrne J. \& Conroy R. (2002) The SAVI Report - Sexual Abuse and Violence in Ireland: A National Study of Irish Experiences, Beliefs and Attitudes Concerning Sexual Violence. Liffey Press, Dublin.

Monnier J., Resnick H.S., Kilpatrick D.G. \& Seals B. (2002) The relationship between distress and resource loss following rape. Violence and Victims 17 (1), 85-92.

O'Shea A. (2006) Sexual Assault Treatment Services: A National Review. National Steering Committee on Violence Against Women, Dublin.

O'Sullivan E. \& Carlton A. (2001) Victim services, community outreach, and contemporary rape crisis centres: a comparison of independent and multi-service centres. Journal of Interpersonal Violence 16 (4), 343-360. 
Patton M.Q. (2002) Qualitative Research and Evaluative Methods, 3rd edn. Sage Publications, Thousand Oaks, CA.

Petrak J. \& Hedge B. (2002) The Trauma of Sexual Assault: Treatment, Prevention and Practice. Wiley \& Sons, West Sussex.

RCNI (2005) Agenda for Justice: Towards Ending Injustice for Survivors of Sexual Violence. Rape Crisis Network Ireland, Dublin.

Regan L. \& Kelly L. (2003) Rape: Still a Forgotten Issue. Child and Woman Abuse Studies Unit, London Metropolitan University, London.

Renner K.E., Wackett C. \& Ganderton S. (1988) The 'social' nature of sexual assault. Canadian Psychology 29 (2), 163 173.

Sable M.R., Danis F., Mauzy D.L. \& Gallagher S.K. (2006) Barriers to reporting sexual assault for women and men: perspectives of college students. Journal of American College Health 55 (3), 157-162.

Schumm J.A., Hobfoll S.E. \& Keogh N.J. (2004) Revictimization and interpersonal resource loss predicts PTSD among women in substance-use treatment. Journal of Traumatic Stress 17 (2), 173-181.

Stein M.B., Lang A.J., Laffaye C., Satz L.E. \& Dresselhaus T.R. (2004) Relationship of sexual assault history to somatic symptoms and health anxiety in women. General Hospital Psychiatry 26 (3), 178-183.

Tavara L. (2006) Sexual violence. Best Practice \& Research: Clinical Obstetrics \& Gynaecology 20 (3), 395-408.

Tjaden P. \& Thoennes N. (2006) Extent, Nature and Consequences of Intimate Partner Violence: Findings from the National Violence Against Women Survey. Special Report. National Institute of Justice, Office of Justice Programs, U.S. Department of Justice, Washington, D.C.

Ullman S.E. (2004) Sexual assault victimization and suicidal behaviour in women: a review of the literature. Aggression and Violent Behaviour 9 (4), 331-351.

Women's Health Council (2007) Violence Against Women and Health. Women's Health Council, Dublin.

Young P., O'Donnell I. \& Clare E. (2001) Crime in Ireland: Trends and Patterns 1950-98. The National Crime Council, Dublin. 